\title{
Association of Patient Language with Guideline-Concordant Care for Individuals with Chronic Kidney Disease (CKD) in Primary Care
}

\author{
Sarah Rosenwohl-Mack, MPH ${ }^{7}$, Anna Rubinsky, $P h D^{1,2}$, Leah Karliner, $M D^{7}$, \\ Lenny López, $M D^{7}$, and Carmen A. Peralta, $M D^{1,2,3}$ \\ 'University of California, San Francisco, San Francisco, CA, USA; ${ }^{2}$ Kidney Health Research Collaborative, University of California, San Francisco, CA, \\ USA; ${ }^{3}$ Cricket Health, Inc., San Francisco, CA, USA.
}

J Gen Intern Med 34(11):2348-50

DOI: $10.1007 / \mathrm{s} 11606-019-05113-4$

(c) Society of General Internal Medicine 2019

\section{INTRODUCTION}

Adequate primary care has the potential to reduce the high morbidity among persons with chronic kidney disease (CKD). ${ }^{1}$ However, major gaps in care are documented, and these gaps are larger among race/ethnic minority groups, compared with whites. ${ }^{2}$ Whether a patient's level of English proficiency and language preference contributes to gaps in appropriate CKD care prior to kidney failure remains poorly understood. ${ }^{3}$ Persons with limited English proficiency (LEP) are less likely than English speakers to receive optimal care, independent of selfreported race/ethnicity. ${ }^{4}$ We evaluated the association of non-English language preference with guidelineconcordant CKD care among adults with low eGFR who had active primary care in a well-resourced clinic with easy access to multimodal medical interpretation.

\section{METHODS}

We used University of California, San Francisco (UCSF) electronic medical records (EMR) data to identify a cohort of persons with CKD in primary care as previously described. ${ }^{5}$ We defined CKD as two eGFR measurements between 15 and $<60 \mathrm{ml} / \mathrm{min} / 1.73 \mathrm{~m}^{2}$ at least 3 months apart. ${ }^{6}$ This study was approved by the institutional review board at UCSF.

Patients were considered non-English language preferring if the EMR documented preferred language was not English. We considered providers and patients to be language-concordant if records indicated that the provider spoke the non-English language preferred by the patient.

Outcomes of interest were evidence-based processes of care from international guidelines: ${ }^{6}$ (1) testing for albuminuria, which is required for risk stratification; (2) testing for hemoglobin A1c for diabetics; (3) prescription of inhibitors of the renin-angiotensin system (ACEi/ARB) for patients with diabetes or hypertension; (4) prescription

Published online August 26, 2019 of a statin for patients age $>50$ years; (5) BP $<140$ / $90 \mathrm{mmHg}$; and (6) hemoglobin A1c $<7$ for patients with diabetes, during the study period. Covariates included patient demographics, comorbidities, insurance type, and number of primary care visits, as previously described. ${ }^{5}$

We compared characteristics by patient language preference (English vs. non-English) using chi-squared tests. We estimated the relative risk of each outcome for patients with non-English language preference compared with English-language preference using multivariable modified Poisson regression models with logarithm link, clustered on provider and adjusting for potential confounders. Finally, we stratified analyses by provider-patient language concordance.

\section{RESULTS}

Among 1726 persons with CKD, 17\% preferred a language other than English. The most common language preferred was Cantonese (30\%), followed by Spanish (14\%), Russian (13\%), Vietnamese (10\%), Mandarin (8\%), and Korean (7\%). Compared with English preference, those with a non-English preference were older, had more comorbidities, were more likely to have Medicare, and had higher number of primary care visits (Table 1).

Approximately half (49\%) of the providers reported speaking a language in addition to English, and patient and provider language were considered concordant for $89 \%$ of patients.

After adjusting for age and sex, there was a $34 \%$ higher rate of albuminuria testing among those with non-English preference, but differences were attenuated after adjustment for other covariates (RR $1.03(0.90,1.17))$. Compared with patients with English preference, those with non-English preference had a $13 \%$ higher rate of a statin prescription after adjustment for age and sex, and this difference was attenuated after adjustment for other covariates (RR $1.01(0.94,1.09))$. We found no differences by language preference for other comparisons ( $p$ values $\geq$ 0.2 ). Results did not vary when stratified by providerpatient language concordance $(p>0.05)$. 
Table 1 Characteristics of 1726 Patients with eGFR-Based CKD Who Have Active PCP Follow-up by Patient Language Preference

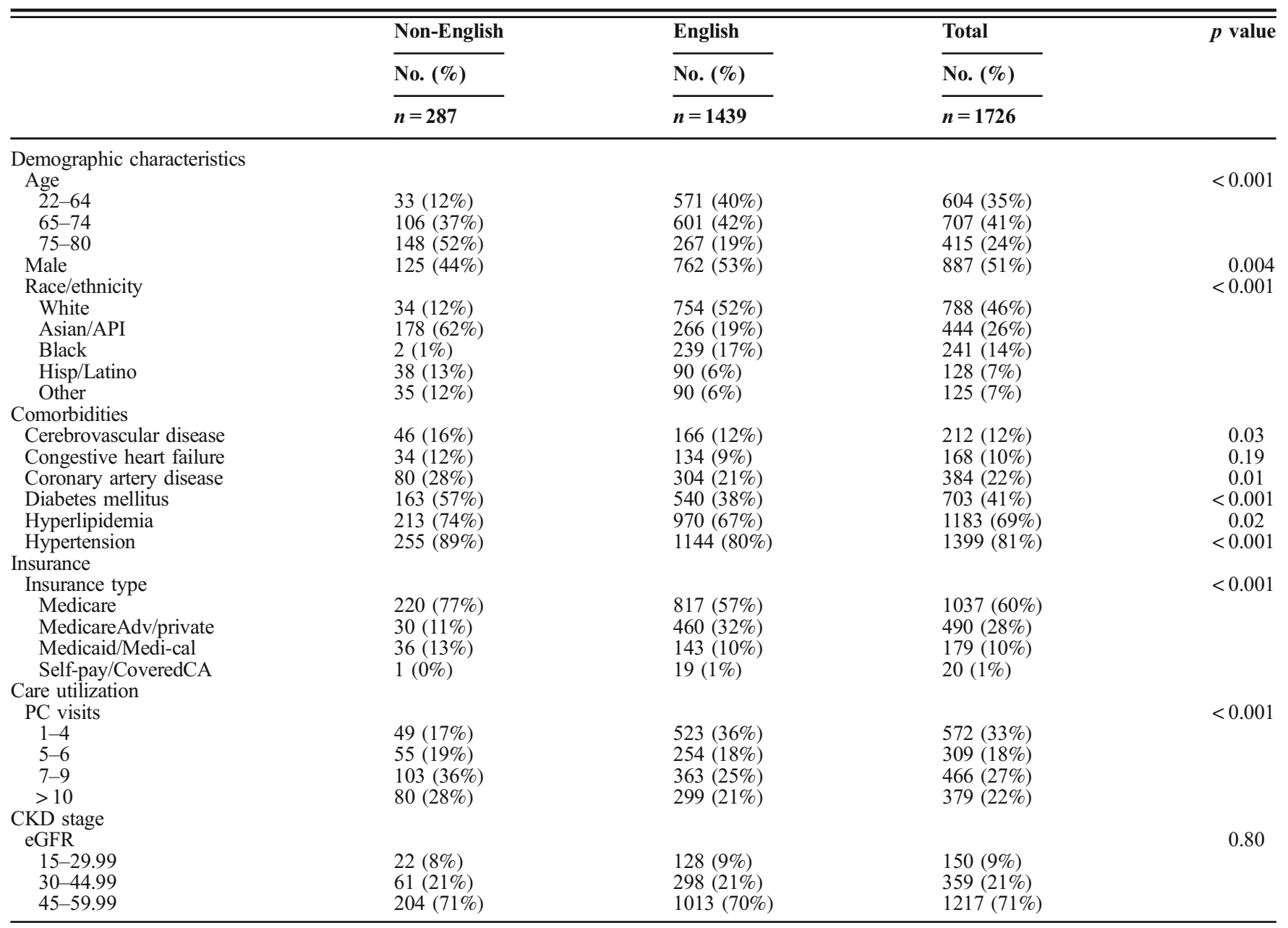

Table 2 The Association of Patient Language Preference with CKD Processes of Care

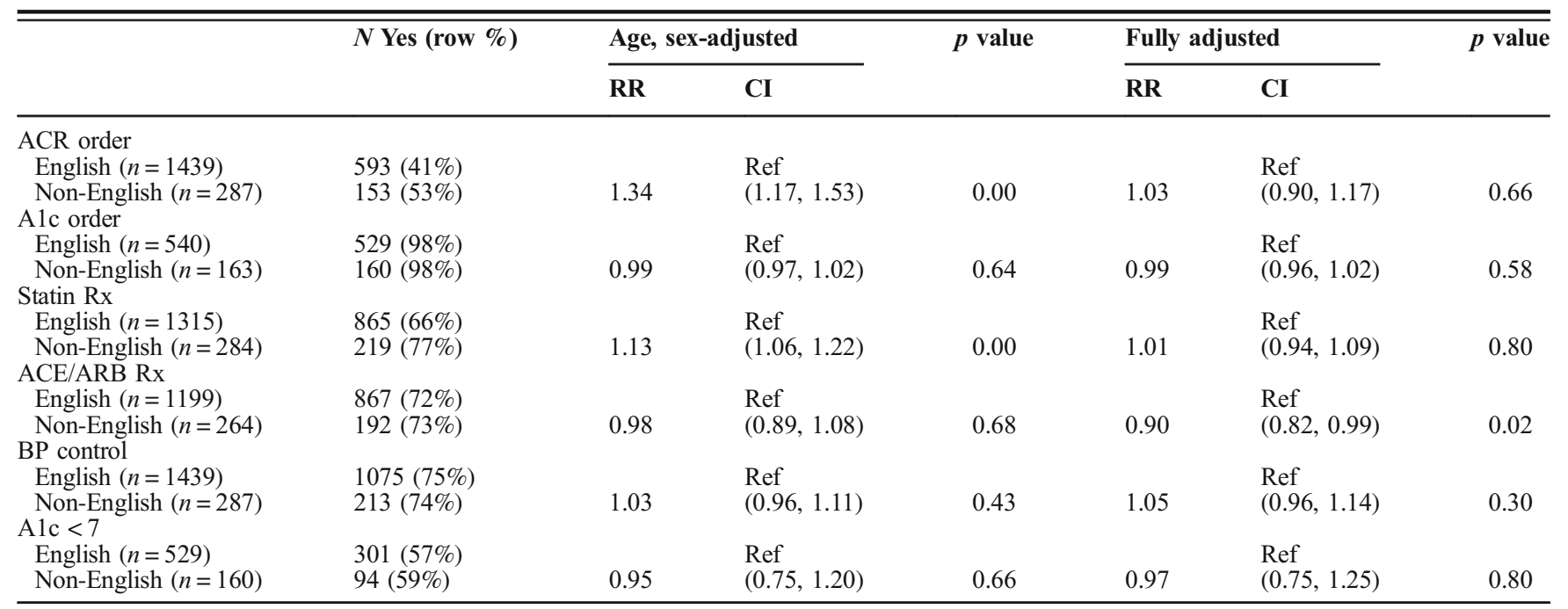

Age, sex-adjusted: adjusted for age, sex only; clustered on provider $(n=152)$

Fully adjusted: additionally adjusted for racelethnicity, insurance type, number of PC visits, CKD stage, and count of comorbid conditions 


\section{DISCUSSION AND CONCLUSIONS}

We found that language preference was not significantly associated with rates of guideline-concordant processes of care for CKD in a well-resourced primary care clinic with universal access to interpreters (Table 2). Moreover, considering language concordance between patient and provider did not change our findings.

A strength of this study is its novelty and relatively large and diverse sample. Limitations include use of EMR data which may lead to misclassification in ascertainment for study variables. In addition, we cannot determine if an interpreter was present at every appointment.

This study adds important information to our understanding of differences in CKD care for persons of under-represented groups and low socioeconomic status. ${ }^{2}$ Specifically, in a setting in which all patients have insurance, consistent access to a primary care, and universal access to professional interpreters, equity of care is achievable. While guideline-concordant care may not perfectly correlate with improved clinical outcomes, our findings highlight the importance of investing in deploying well-resourced primary care for diverse populations.

Corresponding Author: Sarah Anna Leah Lenny Rosenwohl-Mack Rubinsky Karliner López, MPH; Kidney Health Research Collaborative University of California, San Francisco and San Francisco VA, Cricket Health, Inc., San Francisco, CA, USA

(e-mail: Carmenalicia.peralta@ucsf.edu).
Funding Information The authors received funding from the following grants: AHA: 17IEA33410161; R18: 1R18DK110959-01

\section{Compliance with Ethical Standards:}

This study was approved by the institutional review board at UCSF.

Conflict of Interest: Carmen A. Peralta is listed as a chief of medical officer for Cricket Health, Inc.

\section{REFERENCES}

1. Go AS, Chertow GM, Fan D, McCulloch CE, Hsu C. Chronic kidney disease and the risks of death, cardiovascular events, and hospitalization. N Engl J Med 2004;351(13):1296-1305. https://doi.org/10.1056/ NEJMoa041031

2. Norton JM, Moxey-Mims MM, Eggers PW, et al. Social Determinants of Racial Disparities in CKD. J Am Soc Nephrol 2016;27(9):2576-2595. https://doi.org/10.1681/ASN.2016010027

3. Escobedo W, Weismuller $\mathbf{P}$. Assessing health literacy in renal failure and kidney transplant patients. Prog Transplant Aliso Viejo Calif 2013;23(1):47-54. https://doi.org/10.7182/pit2013473

4. Pippins JR, Alegria M, Haas JS. Association between language proficiency and the quality of primary care among a national sample of insured Latinos. Med Care 2007;45(11):1020-1025. https://doi.org/10.1097/ MLR.Ob013e31814847be

5. Frigaard M, Rubinsky A, Lowell L, et al. Validating laboratory defined chronic kidney disease in the electronic health record for patients in primary care. BMC Nephrol 2019;20(1):3. https://doi.org/10.1186/ s12882-018-1156-2

6. Levin A, Stevens PE, Bilous RW, et al. Kidney disease: Improving global outcomes (KDIGO) CKD work group. KDIGO 2012 clinical practice guideline for the evaluation and management of chronic kidney disease. Kidney Int Suppl 2013;3(1):1-150. https://doi.org/10.1038/kisup.2012.73

Publisher's Note Springer Nature remains neutral with regard to jurisdictional claims in published maps and institutional affiliations. 